\title{
GOOD THINGS DO NOT HAPPEN TO ME... BUT NEITHER DO THE BAD THINGS: COMPARATIVE OPTIMISM AND PESSIMISM IN ASLOVAK SAMPLE
}

\author{
Vladimíra ČAVOJOVÁ \\ Institute of Experimental Psychology \\ Center of Social and Psychological Sciences, Slovak Academy of Sciences \\ Dúbravská cesta 9,84114 Bratislava, Slovak Republic \\ E-mail: vladimira.cavojova@savba.sk
}

\begin{abstract}
This study examined whether participants will show optimism about common events, yet show pessimism about rare events (regardless of their desirability), and whether there is a relationship between optimism and overconfidence, conceptualized (Shepperd et al., 2013) as unrealistic absolute optimism. 136 pedagogy students completed a questionnaire with 28 events (positive and negative, rare and common) together with two cognitive tasks and an estimation of their performance. The results support neither the unrealistic hypothesis nor the egocentrism hypothesis fully - the participants appeared to be somewhat pessimistic in estimating the likelihood of mainly positive events happening to them; they were quite optimistic in expecting to avoid negative events. Only a small overlap between the unrealistic comparative optimism and unrealistic absolute optimism (overconfidence) was found. These results support the necessity to distinguish between distinct types of optimism bias and highlight methodological problems connected mainly with estimates of unrealistic comparative optimism.
\end{abstract}

Key words: unrealistic comparative optimism, unrealistic absolute optimism, overconfidence, overestimation, intelligence

The notion that people are frequently overoptimistic about the future is well known in the judgment and decision-making literature (Armor \& Taylor, 2002; Hardman, 2009; Cham-

\section{Acknowledgment}

This work was supported by the Slovak Research and Development Agency under the contract No. APVV-0361-12. I wish to thank my colleagues for their support and comments made on earlier versions of the manuscript. My special thanks go to Miroslav Sirota for his helpful suggestions and to Catriona Menzies for her help with the language and her inspiring questions and comments regarding the manuscript. I also want to thank Marek Jurkovič for help with statistical analyses. bers \& Windschitl, 2004; Kruger \& Burrus, 2004; Lench \& Ditto, 2008; Menon, Kyung, \& Agrawal, 2009; Sharot, 2011; Sweldens, Puntoni, Paolacci, \& Vissers, 2014; Weinstein, 1980; White, Cunningham, \& Titchener, 2011). However, the concept of unrealistic optimism has been questioned recently, both on the basis of terminological confusions and methodological problems (e.g., Harris \& Hahn, 2011). Shepperd, Klein, Waters, and Weinstein (2013) address some of the raised criticisms in their review and propose to distinguish between four kinds of unrealistic optimism. In this paper I build on the distinction made by Shepperd et al.

DOI: $10.21909 / \mathrm{sp} .2016 .04 .724$ 
(2013) and examine the overlap between unrealistic comparative optimism and overconfidence, which can be conceptualized as unrealistic absolute optimism at the individual level according to Shepperd et al. (2013). The aim of this paper, therefore, is to contribute to the debate by empirically examining comparative optimism and overconfidence and the mutual overlap between these concepts. To achieve this aim I replicated the study of Kruger and Burrus (2004) on a sample of Slovak students. Before proceeding to the description of the research, I will first clarify the terminology used in this paper and possible connections between the concepts discussed.

\section{What is Meant by Optimism?}

One of the first researchers who experimentally demonstrated optimistic bias was Weinstein (1980). He showed that majority of participants believed that negative events are less likely while positive events as more likely to happen to them. Later, Scheier and Carver (1985) observed individual differences in the generalized expectancies and they designed the Life Orientation Test (LOT) to measure this kind of dispositional optimism, which was defined as global expectancy of more positive than negative events in one's life. Whether situational or dispositional, scientific definitions of optimism and pessimism tend to focus on expectancies for the future (Carver, Scheier, \& Segerstrom, 2010). According to Carver, Scheier, and Segerstrom (2010) these definitions are linked to expectancy-value models of motivation, which assume that behavior reflects the pursuit of goals. Pursuit of goals is affected by value of the goal and the expectancy (confidence that the goal can be attained). Thus, "optimism and pessimism are broad, generalized versions of confidence and doubt; they are confidence and doubt pertaining to life, rather than to just specific context (Scheier \& Carver, 1992). Thus optimists should tend to be confident and persistent in the face of diverse life challenges... Pessimists should be doubtful and hesitant in the same situations" (Carver et al., 2010, p. 880).

Prevalence of findings that people are generally overly optimistic about their future prospects and that they judge themselves as better on many desirable traits lead Taylor and Brown $(1994,1988)$ to formulate a positive illusions hypothesis - that unrealistic optimism, overly positive self-evaluation and exaggerated perceptions of control and mastery are characteristic of normal mental thought and even more, that they actually promote mental health and ability to engage in productive work and social relationships. Despite the appeal of the positive illusion hypothesis, the notion that biased selfimage and ungrounded beliefs can be beneficial to mental health was heavily criticized by many researchers (Colvin \& Block, 1994; Colvin, Funder, \& Block, 1995) ${ }^{1}$.

\footnotetext{
${ }^{1}$ Reconciliations of the opposing findings by Taylor and Brown and their critiques (Asendorpf \& Ostendorf, 1998) concern methodological issues related to the self-enhancement part of positive illusions and is beyond the scope of this article. However, despite some evidence that positive illusions can have some benefits for some people, Correia (n.d.) argues that such benefits tend to be short-lived and that misbeliefs are generally not adaptive from a long-term perspective. Positive illusions may give some rewarding vision of self, but in the long-time perspective they undermine the process of deliberation and are ultimately detrimental to the maximization of people's preferences.
} 
However, the debate between researchers advocating a positive role of biases and those who favor a perspective more in line with reality is further complicated by conceptual, methodological and terminological issues. For example, people display unrealistic optimism when judging their vulnerability to negative events or the prospect of a positive event occurring (Eiser, Pahl, \& Prins, 2001), but they often also show optimism when comparing themselves with other people (Eiser et al., 2001; Sweldens et al., 2014). This tendency is often considered as an expression of the above-average-effect or self-serving bias. However, people sometimes display pessimism as well, when comparing their prospects with other people (Kruger \& Burrus, 2004), especially when frequency of the event is taken into account. Various terms have been used interchangeably to describe this kind of optimistic bias, such as unrealistic optimism, planning fallacy, illusion of control, better-than-averageeffect and overconfidence, sometimes having synonymous and sometimes related meaning (Harris \& Hahn, 2011). Shepperd, Carroll, Grace, and Terry (2002) argue that people evaluate their future success usually in comparison with other people. Comparative optimism is then defined as a belief that one is less likely to experience a negative event and more likely to experience a positive event than other people are, whereas comparative pessimism is a belief that one is less likely to experience a positive event and more likely to experience negative event than other people are.

In recent review Shepperd et al. (2013) identified four types of optimism: unrealistic absolute optimism at the individual and group level and unrealistic comparative optimism at the individual and group level.
Unrealistic absolute optimism refers to the idea that the expectation is unrealistic and optimistic relative to an objective standard, whereas unrealistic comparative optimism refers to the idea that expectation is unrealistic and optimistic relative to the estimates a person makes for other people. Moreover, they argue that many of criticisms towards the concept of optimism bias suggesting that the optimism bias is a mere statistical artefact, relates only to one of the types: the unrealistic comparative optimism at group level.

The approach of Shepperd et al. (2013) seems to be very useful for systemizing research findings related to optimism and in recognizing new areas of research, such as conceptual distinctions among types of unrealistic optimism. They suggest that unrealistic absolute optimism and unrealistic comparative optimism may reflect different underlying constructs. However, the ways they describe unrealistic absolute optimism at individual level seem to overlap to a great extent with another related construct - overconfidence. Although Shepperd's et al. (2013) definition pertains to assessing risks related to some external objective standard, the examples they give and research they review is at least in some way related to estimating one's own performance (such as grades).

\section{Comparative Optimism and Overconfidence}

Some researchers (Moore \& Healy, 2008; Olsson, 2014) observed a lack of conceptual clarity in the overconfidence literature as well. As shown in the review about optimism, sometimes optimism is confused with overconfidence, or confidence is seen as a part of optimistic disposition (Carver et al., 2010). 
Overconfidence, too, has been studied inconsistently and it is possible to describe three kinds of overconfidence (Moore \& Healy, 2008): 1) Overestimation refers to predicting that one's performance in a given task will be better than it actually is; 2) overplacement refers to evaluating one's performance more positively relative to others; 3 ) overprecision refers to excessive certainty in one's beliefs and estimates. Overplacement is most directly related to comparisons with other people, thus, it is probably most associated with comparative optimism. And while Shepperd et al. (2013) define unrealistic optimism in terms of risk estimates, it was clear from their presentation that they consider some instances of overestimation as manifestation of unrealistic absolute optimism at individual level.

However, only a few studies have examined the relationships between these similar concepts and the way they are measured (Larrick, Burson, \& Soll, 2007; Moore \& Healy, 2008; Radcliffe \& Klein, 2002). Shepperd et al. (2013) call for more research that would help clarify the question whether the four types of unrealistic optimism are really conceptually different or they merely differ because of different measurements, but otherwise represent a single underlying construct.

They suggest that at least unrealistic absolute optimism and unrealistic comparative optimism reflect a different underlying construct. Knowing whether there are conceptual differences between the types of optimism is important, because different types of optimism can have different causes and different consequences, and can be elicited by different situational determinants.

For example, some studies have shown that unrealistic optimism is not associated with low-risk perception (Wiebe \& Black, 1997, in Radcliffe \& Klein, 2002), suggesting that unrealistic optimists use defensive strategies to avoid updating risk information. Radcliffe and Klein (2002) found that dispositional optimism was correlated with unrealistic comparative optimism, but not with unrealistic absolute optimism. In their study, dispositional optimists and people giving lower comparative risk perceptions (irrespective of their accuracy) had lower risk of having a heart attack. In other words, Radcliffe and Klein (2002) showed that "believing one's risk to be below average is not maladaptive; on the contrary, it seems to be a fairly accurate belief that is associated with a variety of favorable outcomes" (p. 844). This was not the case for unrealistic optimism - these individuals were at higher risk, yet believed they were at lower risk.

Radcliffe and Klein's (2002) study highlighted the importance of distinguishing between adaptive (comparative) and maladaptive (absolute) optimism and their differentiated correlation with related issues, such as risk perception. Unlike other studies, they used more objective estimates for unrealistic optimism at the individual level (Health Risk Appraisal), but they focused only on a negative life event (risk of heart attack).

Similarly, Moore and Healy (2008) call for the need to distinguish three distinct ways of conceptualizing and measuring overconfidence. Although both these studies attempted to look for similarities and differences between differently conceptualized optimism and overconfidence, they did not look at the mutual relationships. Only, Larrick et al. (2007) examined the relationship between social comparison bias (better-than-average effect) and overconfidence (over-precision), and they found that higher 
perceptions of one's ability relative to others predicted greater degrees of overconfidence.

\section{Cultural Influences on Optimism}

One of the critiques regarding prevalence and normalcy of optimism bias and overconfidence is based on the fact that majority of studies are based on Western, mainly U.S. populations, which undermine the generalizability of the findings (Henrich, Heine, $\&$ Norenzayan, 2010). Some authors (Norem \& Chang, 2002) argue that due to the dominancy of American culture, people (and scientists as well) may underestimate the costs of optimism. There has been some research showing greater optimism and overconfidence in Western, more individualistic cultures (Heine \& Lehman, 1995; Heine, 1993; Chang, 1996), but in most cases North American cultures (Canada, USA) were compared with Asian cultures. Heine and Lehman (1995), using comparative measure similar to those of Weinstein, found that Canadians showed significantly more optimism than Japanese and it was more strongly related to perceived threat. Chang (1996) compared Asian Americans and Caucasian Americans in dispositional optimism and again found significantly greater optimism in Caucasian Americans. However, meta-analysis of dispositional optimism (Fischer \& Chalmers, 2008) using data from 22 nations found that overall effects of culture are generally small. Although, greater individualism was associated with greater optimism; claims of fundamental cultural differences were not supported. It should be noted, however, that Hofstede's estimations of cultural dimensions were recently questioned (Bašnáková, Brezina, \& Masaryk, 2016).
For the purpose of the current paper, the study by Rose, Endo, Windschitl, and Suls (2008) is of special interest, because it also examines possible methodological effects in the study of cultural differences. They compared direct (a single comparison judgment between self and peers) and indirect method (separate self and peer judgments). They found that a direct method produced similar patterns across cultures - i.e., unrealistic optimism about experiencing infrequent/ negative events but pessimism about experiencing frequent/negative events. On the other hand, an indirect method produced different patterns across cultures - i.e., U.S. sample was more unrealistically optimistic than the Japanese sample.

However, it is also important to study the differences within the so-called Western cultures, and the countries of Eastern Europe are of special interest, as they are usually considered to be more collectivistic than traditional Western democracies (Bašnáková et al., 2016; Kolman, Noorderhaven, Hofstede, \& Dienes, 2003).

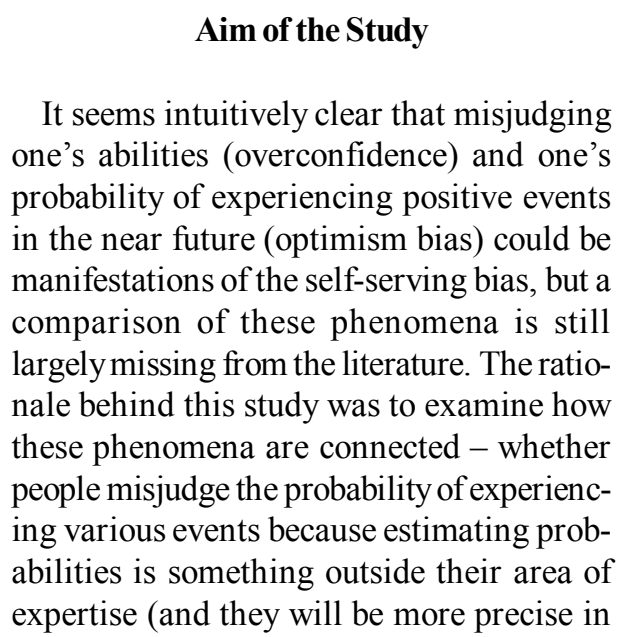


estimating their own abilities, which they are more familiar with) or they misjudge the probabilities and their abilities due to the same underlying factor, such as self-serving bias. In other words, are the various expressions (types) of optimism (as defined also by Shepperd et al., 2013) manifestations of one underlying construct or are they distinct concepts?

Therefore, the following hypotheses and research questions were tested. Firstly, I focused on verifying the results of Kruger and Burrus (2004) on non-Western sample (Henrich et al., 2010): 1) Participants will show optimism for common events (regardless of their desirability), but will show pessimism for rate events (regardless of their desirability). Then, the relationship between optimism and overconfidence will be tested: 2) The participants who will show higher optimism will also show higher overconfidence. After testing these two main hypotheses, secondary analyses will focus on the comparison, 3) whether direct and indirect measure of optimism will yield different results due to lower egocentrism using indirect measure (Rose et al., 2008).

\section{Method}

\section{Participants}

Overall, 136 pedagogy students of various majors from Constantine the Philosopher University in Slovakia participated in the study: 109 of them participated in the first session; 121 of them participated in the second session, giving us a complete dataset from 92 students who participated in both sessions. The mean age of our sample was $22.04(S D=4.45)$ years and was similar to the study by Kruger and Burrus (2004) in terms of age and probable motivation (they were given extra course credits), but because the sample was drawn from the pool of pedagogy students, it consisted predominately of women $(83.1 \%)$.

\section{Materials}

Unrealistic Comparative Optimism: Experiencing Rare vs. Common Events Questionnaire

Participants $(N=109)$ completed a questionnaire describing 28 events (we used almost the same ${ }^{2}$ material as that in Kruger \& Burrus, 2004, Study 1). The wording of the items used can be seen in Table 3 in the Results section. The rationale for using Kruger and Burrus's (2004) material was to replicate the main effect of the frequency of the event, rather than its desirability. The questionnaire balances the frequency (common vs. rare) and desirability (positive vs. negative) of the event, and at the same time it uses events

\footnotetext{
${ }^{2}$ The exact same events were used, with only four exceptions. 1) I changed the starting salary to $€ 800$ in the common positive group and $€ 8000$ in the rare positive group, as the original amounts (\$2500 and \$25000, respectively) would have been too unrealistic in the Slovak setting. 2) Also, pounds were changed to kilos and to highlight the difference between the two conditions (common negative vs. rare negative) the time period in common negative was changed from 10 to 5 years. 3) We omitted one item and used another from Experiment 2 instead, as falling behind with dental hygiene would probably have no effect on our participants. Instead, we used items related to being stressed before an exam and being stressed before the presidential elections. 4) Lastly, we slightly adapted the item regarding graduating in the upper half of one's class into graduating with "honors", which is more natural in a Slovak setting.
} 
that are relatively neutral in terms of social desirability or health-related issues. For each event, participants compared their likelihood of experiencing the event in comparison with the average person from their course on a scale of 1 (much less likely to happen to me) to 7 (much more likely to happen to me). Next, participants estimated the likelihood of the event happening to them and (separately) of it happening to the average person in their class on a scale from 1 (very unlikely) to 7 (very likely). Of the 28 events, half were positive and half were negative, while half were common and half were rare (for a total of 7 events per category). Following Kruger and Burrus (2004) I calculated mean likelihood estimates by subtracting 4 (the midpoint of the scale corresponding to the average) from each estimate and this was our main outcome variable (direct measure of comparative optimism). I also calculated the indirect measure of comparative optimism by subtracting peer-estimates from self-estimates. Positive values in all cases indicate that a person has a greater-than-average perceived likelihood of experiencing the event, while negative values indicated that a person has a less-than-average likelihood of experiencing the event.

Overconfidence: Estimating One's Achievement in Cognitively Challenging Tasks

To estimate overconfidence, I used the Vienna Matrix Test (VMT, standardized for the Slovak population by Klose, Černochová, \& Král, 2002) for measuring intelligence and the Cognitive Reflection Test (Frederick, 2005), which are described later in the section in more detail. The choice of cognitive tasks was driven mainly by my expectation that one's own cognitive abilities are personally important, because they carry implicit value and can relate to person's self-esteem and, therefore, people are motivated to monitor their cognitive abilities. Moreover, in school setting students receive frequent feedback about their cognitive abilities in forms of grades. In line with recommendations in overconfidence literature, participants were given some information about tests beforehand, e.g., scores in VMT that represent below-average, average, and above-average intelligence and nature of logical tasks (CRT).

Vienna Matrix Test (VMT). The VMT is based on Raven's classic test of progressive matrices - two items are taken from the Standard Progressive Matrices and one from the Advanced Progressive Matrices constructed by Raven. It consists of 24 items of increasing difficulty and it is time-limited ( 25 minutes). Each task contains a $3 \times 3$ picture matrix where the picture is missing in the third row. The participant's task is to correctly fill in one of the eight possibilities. The VMT shows high correlations with the Intelligence Structure Test and Klose et al. (2002) conclude that it reliably reflects general cognitive factors. The test is supposed to be culturally fair as it is based on figural content. The means score for our sample $(N=121)$ was $97.09(S D=$ 16.3); on average the participants solved 15.32 tasks out of 24. Prior to filling in the test, participants were asked to estimate their IQ based on the provided ranges and information about the test. Overconfidence was calculated by subtracting the actual IQ from the expected IQ, thus higher score reflects higher overconfidence and negative score reflects underconfidence (actual score was higher than expected). 
Cognitive Reflection Test-expanded version (Toplak, West, \& Stanovich, 2014). The CRT is a simple test measuring a person's tendency to process things poorly (or cognitive impulsiveness/laziness in defaulting to the autonomous mind) against a person's ability to postpone judgment (cognitive reflection). It is a simple test of one type of cognitive ability that is so predictive of some preferences that it effectively functions as an expression of cognitive ability (Frederick, 2005). However, because it is so widely used as an example in textbooks, the questions have become widely known, so recently Toplak et al. (2013) expanded the three-task CRT (CRT3) into a seven-task CRT (CRT7). This is the test I used in this study. The scoring remained the same: correct responses were ascribed 1 point resulting in an average $M=0.81$ for CRT 3 and $M=2.01$ for CRT7 in the current study.

Moreover, participants made three kinds of estimations: one before the task (How many of the tasks will you solve correctly?), one after the task (How sure you are that you solved the tasks correctly) and one about overplacement (What percentage of your fellow students do you think did worse than you in this task?). The first question (before the performance) ${ }^{3}$ was used for calculation of overconfidence: the actual score from the estimated score for CRT. A positive number indicated overconfidence (the estimate was higher than actual performance); while negative numbers indicated underconfidence (the estimate was lower than actual performance).

\footnotetext{
${ }^{3}$ Altough usually the question is asked after the performance, in this case it should reflect expectancy about the future performance, which should be more related to the positive expectancy of other future events.
}

Lastly, participants also estimated their course grade. Estimates and actual grades were recoded so that a higher score means a better grade. Overconfidence was again calculated by subtracting the actual grade from the expected grade; however, not all participants in the study were from one course, so the reported number of participants in the analyses differs from the overall number of participants.

\section{Overview of the Procedure}

Participants completed all measures in two sessions online via Survio survey software. The first session, "Predicting future events", consisted of estimating likelihoods of 28 events happening to them in comparison with average student in their class (direct measure), estimating likelihoods of the same 28 events happening to them and then separately happening to the average student in their class (indirect measure). Then they were asked to estimate how many of the following 7 logical tasks will they solve correctly. They completed 7 cognitive tasks, again answered how many tasks they believe they solved correctly and estimated percentage of students that did worse. The measurement of intelligence was done separately and again, before completing the test, participants were asked to estimate their IQ and they were given the range of norms for the test used. Demographic variables were collected at both sessions and these included: gender, age, district, and education level. Descriptive statistics for all measures are presented in Table 1.

\section{Overview of Data Analyses}

First, I used one-way Wilcoxon Signed Ranks Test for analyzing whether estimated 
Table 1 Descriptive statistics for the whole sample

\begin{tabular}{|c|c|c|c|c|}
\hline \multicolumn{5}{|c|}{ Total } \\
\hline Variable & $N$ & $M$ & $S D$ & $95 \% \mathrm{CI}$ \\
\hline Age & 136 & 22.04 & 4.46 & {$[21.28,22.79]$} \\
\hline \multicolumn{5}{|l|}{ Grades } \\
\hline actual & 68 & 2.63 & 1.34 & {$[2.31,2.96]$} \\
\hline estimated & 109 & 2.74 & 1.29 & {$[2.5,2.99]$} \\
\hline overconfidence & 65 & 0.05 & 1.24 & {$[-0.26,0.36]$} \\
\hline \multicolumn{5}{|l|}{ IQ (VMT) } \\
\hline actual & 121 & 97.09 & 16.30 & {$[94.15,100.03]$} \\
\hline estimated & 121 & 103.81 & 10.10 & {$[101.99,105.63]$} \\
\hline overconfidence & 121 & 6.72 & 17.03 & {$[3.88,10.16]$} \\
\hline \multicolumn{5}{|l|}{ CRT7 } \\
\hline actual & 109 & 2.01 & 1.96 & {$[1.64,2.38]$} \\
\hline estimated & 109 & 4.25 & 1.43 & {$[3.98,4.52]$} \\
\hline overconfidence & 109 & 2.24 & 2.06 & {$[1.85,2.63]$} \\
\hline \multicolumn{5}{|l|}{ DM } \\
\hline \multicolumn{5}{|l|}{ Comparative optimism } \\
\hline common negative & 109 & -0.68 & 6.01 & {$[-1.82,0.46]$} \\
\hline rare positive & 109 & -14.15 & 7.78 & {$[-15.62,-12.67]$} \\
\hline rare negative & 109 & -12.29 & 5.50 & {$[-13.34,-11.25]$} \\
\hline \multicolumn{5}{|l|}{ IM } \\
\hline \multicolumn{5}{|c|}{ Comparative optimism } \\
\hline common negative & 109 & -4.28 & 5.66 & {$[-5.35,-3.20]$} \\
\hline rare positive & 109 & -1.07 & 7.27 & {$[-2.45,0.30]$} \\
\hline rare negative & 109 & -4.50 & 5.22 & {$[-5.49,-3.50]$} \\
\hline
\end{tabular}

Note. VMT = Vienna Matrix Test; CRT7 = expanded version of Cognitive Reflection

Test; $\mathrm{DM}=$ Direct measure; $\mathrm{IM}=$ Indirect measure

likelihoods for self(using direct measure) are significantly different from the hypothesized mean $=0$ (which would be the case if participants would estimate the same likelihoods for selfand their peers for all kinds of events). Then I used Wilcoxon Signed Ranks Test for 2 related variables (self estimates vs. peer estimates) for analyzing differences using indirect measure. For testing the prediction that participants will show optimism for common events (regardless of their desirability), but will show pessimism for rate events (regardless of their desirability) two-way Friedman test (as non-parametric alternative to the oneway ANOVA with repeated measures) was performed.

To test whether estimated performance differs from the actual performance the paired t-test was used. To test the hypothesized relationships between optimism and overconfidence I used Pearson correlation. Lastly, to compare whether there were significant shifts in the direction between direct and indirect measure of unrealistic optimism, Wilcoxon Signed Ranks test was used. 


\section{Results}

\section{Frequency versus Desirability of Events}

The first prediction that participants would see themselves as more likely than the average student to experience a common event and less likely than the average student to experience a rare event was tested. Results of direct measure show (Table 2) that this prediction was directionally confirmed in 19 out of 28 cases, and to a statistically significant degree in 18 of them. However, regarding common events there were 7 statistically significant differences in the opposite direction than predicted, i.e. that participants saw themselves as less likely than their peers to experience 4 common positive events (owning a house, work recognized by an award, graduating with honors, and starting salary above $€ 800$ ), and also less likely than their peers to experience 3 common negative events (a speeding ticket, being heartbroken before the age of 40, and being involved in a car accident). Generally, the participants estimated that both positive and negative events are less likely to happen to them than to an average student in their class. Thus, they showed pessimism regarding positive events and optimism regarding negative events (more so for rare negative events). The prediction about the supposed optimism due to the frequency was true only for the rare events. In common events it was true in 5 out of 14 cases. There is the trend in line with the prediction for composite likelihood scores for common positive, rare positive, common negative and rare negative scores (Table 1); moreover, analyzing CIs shows significant differences between the four composite likelihood estimates.
I also analyzed differences between selfestimate and peer-estimate (indirect measure) and in this case, the prediction that participants would see themselves as more likely than the average student to experience a common event and less likely than the average student to experience a rare event was directionally confirmed in 16 out of 28 cases, and to statistically significant degree in 11 of them (Table 3). Again, there were statistical significant differences between estimates of likelihoods for self and other in the opposite direction in 7 cases. Majority of these results opposing the prediction regarded optimism for common negative events, i.e., participants saw themselves as significantly less likely to experience these events.

The means and 95\% confidence intervals for common positive and negative events, and rare positive and negative events are presented in Table 1. They suggest that participants estimated the likelihood of common events happening to them as being much higher than the likelihood of rare events happening to them, compared with the average person and regardless of their desirability. The likelihood of common/rare versus positive/negative events was further analyzed with Friedman test, because the data were not normally distributed.

There was a statistically significant difference in estimated likelihood (direct measure) depending on frequency and desirability of event, $\chi^{2}(3)=175.76, p<.001$. Post-hoc analyses by a Wilcoxon Signed Rank Test with Bonferroni correction set at $p=.0125$ showed that participants displayed significantly larger optimism for rare negative events than common negative events $(Z=-8.723, p=.009)$ and significantly larger pessimism for rare positive events than common positive events $(Z=-8.618, p<.001)$, suggesting sig- 


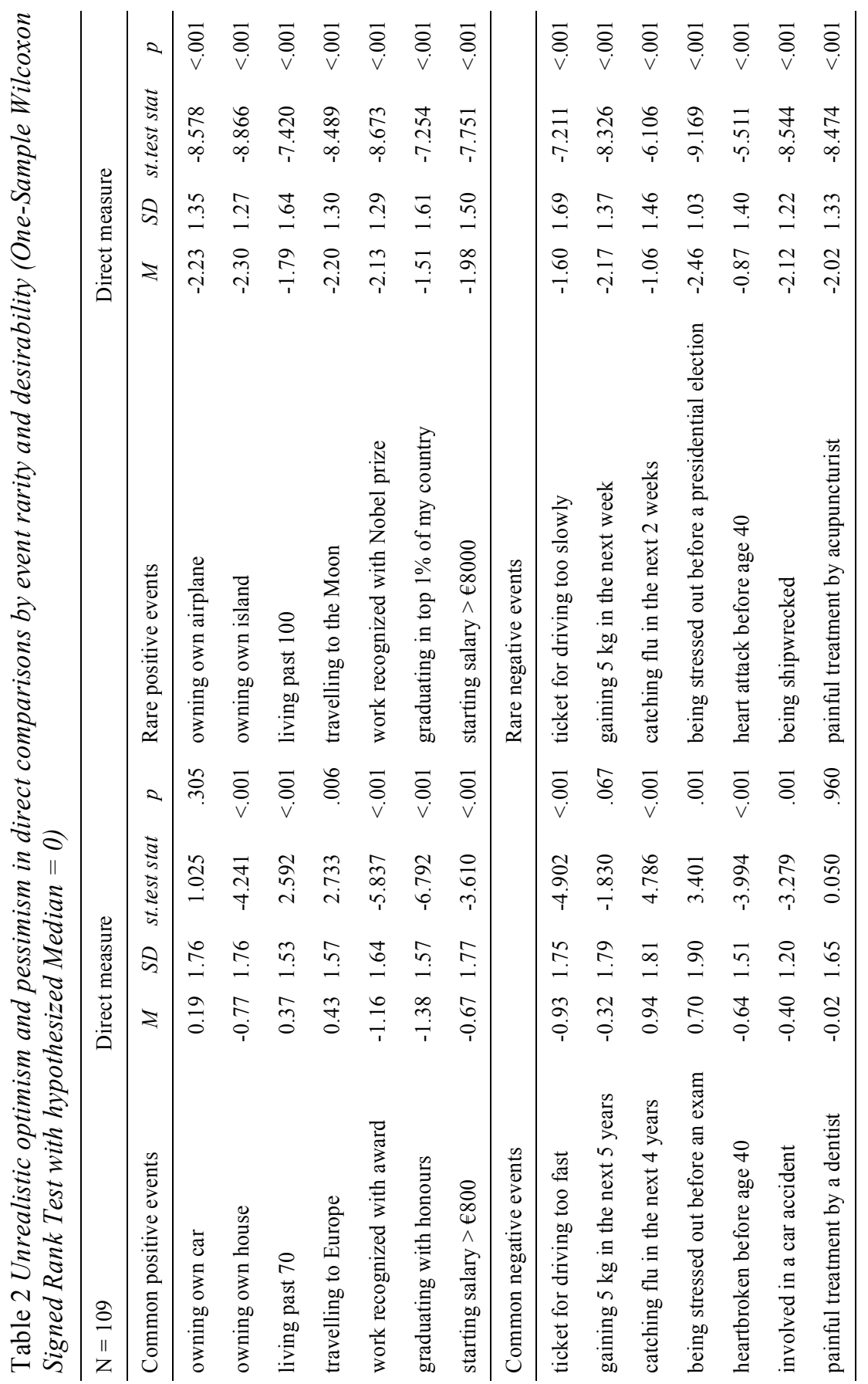




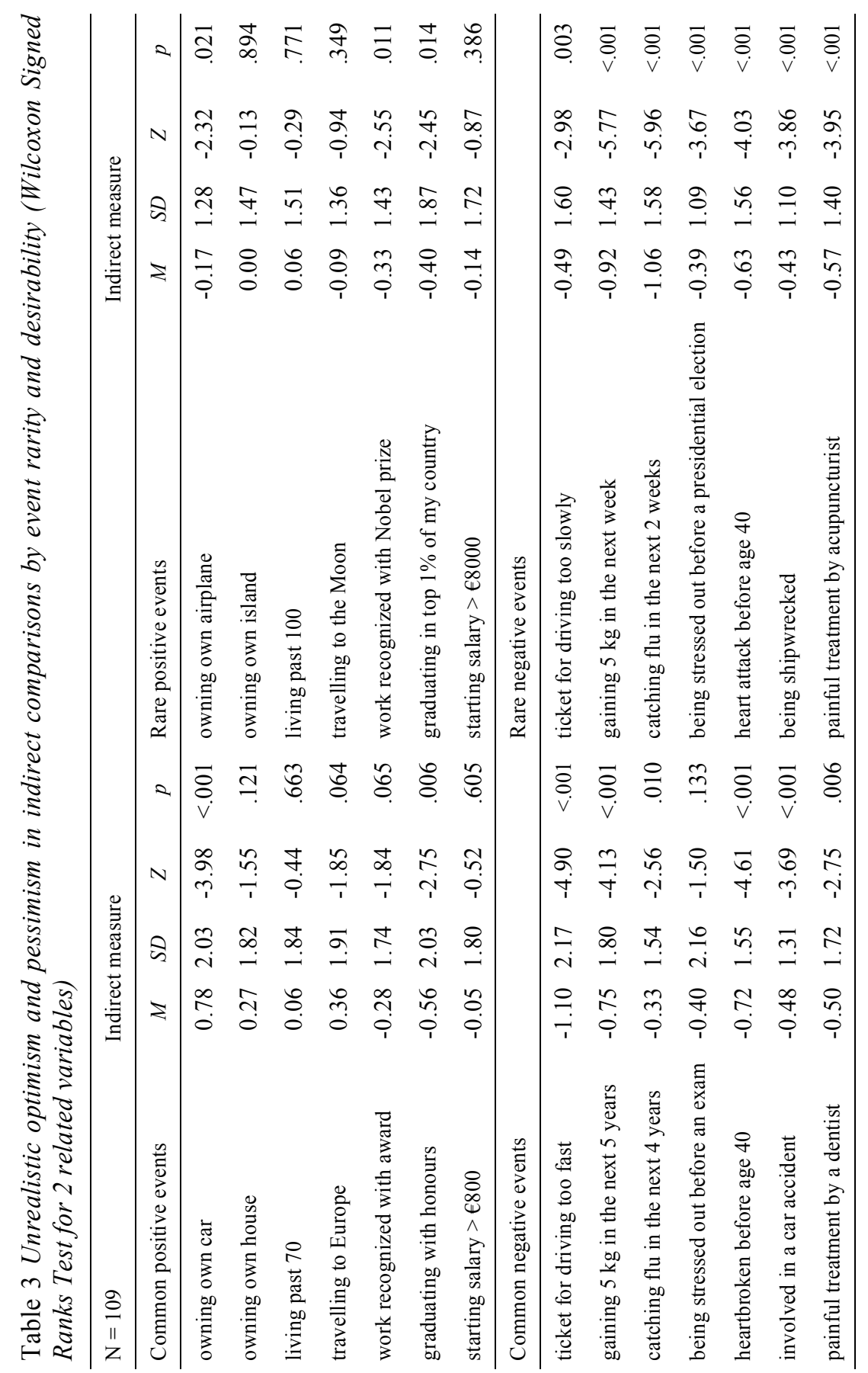


nificant effect of frequency of the event. They also underestimated more the likelihood of common positive than common negative event $(Z=-2.599, p<.001)$, and they underestimated more the likelihood of rare positive than rare negative event $(Z=-3.671, p<$ $.001)$.

The results were then analyzed using indirect measure as well. There was a statistically significant difference in estimated likelihood (indirect measure) depending on frequency and desirability of event, $\chi^{2}(3)=$ $27.893, p<.001$. Post-hoc analyses by a Wilcoxon Signed Rank Test with Bonferroni correction set at $p=.0125$ showed that participants did not display significantly larger optimism for rare negative events than common negative events $(Z=-0.114, p=.909)$ and neither did they display significantly larger pessimism for rare positive events than common positive events $(Z=-2.351, p<.019)$, suggesting there was no significant effect of frequency of the event. However, they underestimated more the likelihood of common negative than common positive event $(Z=-4.778, p<.001)$, and they underestimated more the likelihood of rare negative than rare positive event $(Z=-3.468, p<.001)$, suggesting greater optimism of participants.

Correlations between Optimism/Pessimism and Overconfidence

The participants were also generally overconfident about their performance (Table 1). Their estimated average performance in the CRT was above $1 S D$ more than their actual performance (they expected to correctly solve more than half of the 7 logical tasks, but in fact solved only 2 of the tasks correctly) and this difference was statistically significant $\left(t_{(108)}=28.867, p<0.001\right)$. Their actual IQ was on average 7 points lower than anticipated and again, this difference was statistically significant $\left(t_{(120)}=-4.341, p<\right.$ 0.001 ). Only the grades were estimated with some degree of accuracy by the participants and the difference between the estimated and actual grade was not significant $\left(t_{(64)}=0.299\right.$, $p=0.766$ ).

All three measures of overconfidence correlated weakly together (see Table 4). Generally, there was little correlation between overconfidence on any measure and optimism/ pessimism regarding future events. However, there were the following two correlations: a negative one $(r=-.241, p=.012)$ between overconfidence (CRT) and estimation of the likelihood of common negative events (direct method) and another negative one ( $r=$ $-.259, p=.012)$ between overconfidence (VMT) and estimation of rare negative events (indirect method). In other words, the more overconfident also the more optimistic were the participants (i.e., they estimated that they are less likely than average person to experience common negative or rare negative events).

\section{Comparing Direct and Indirect Measures}

As a last step I performed a set of analyses on the indirect measure of optimism bias. The rationale was that the direct comparisons should involve egocentric overweighting, while indirect comparison can short-circuit the egocentrism by forcing individuals to consider the comparison group (Kruger \& Burrus, 2004; Rose et al., 2008).

The results showed that the difference between self-estimates and peer-estimates in likelihoods of experiencing a given event was smaller when the event was positive $(M($ common $)=0.58, M($ rare $)=-1.07)$ and 


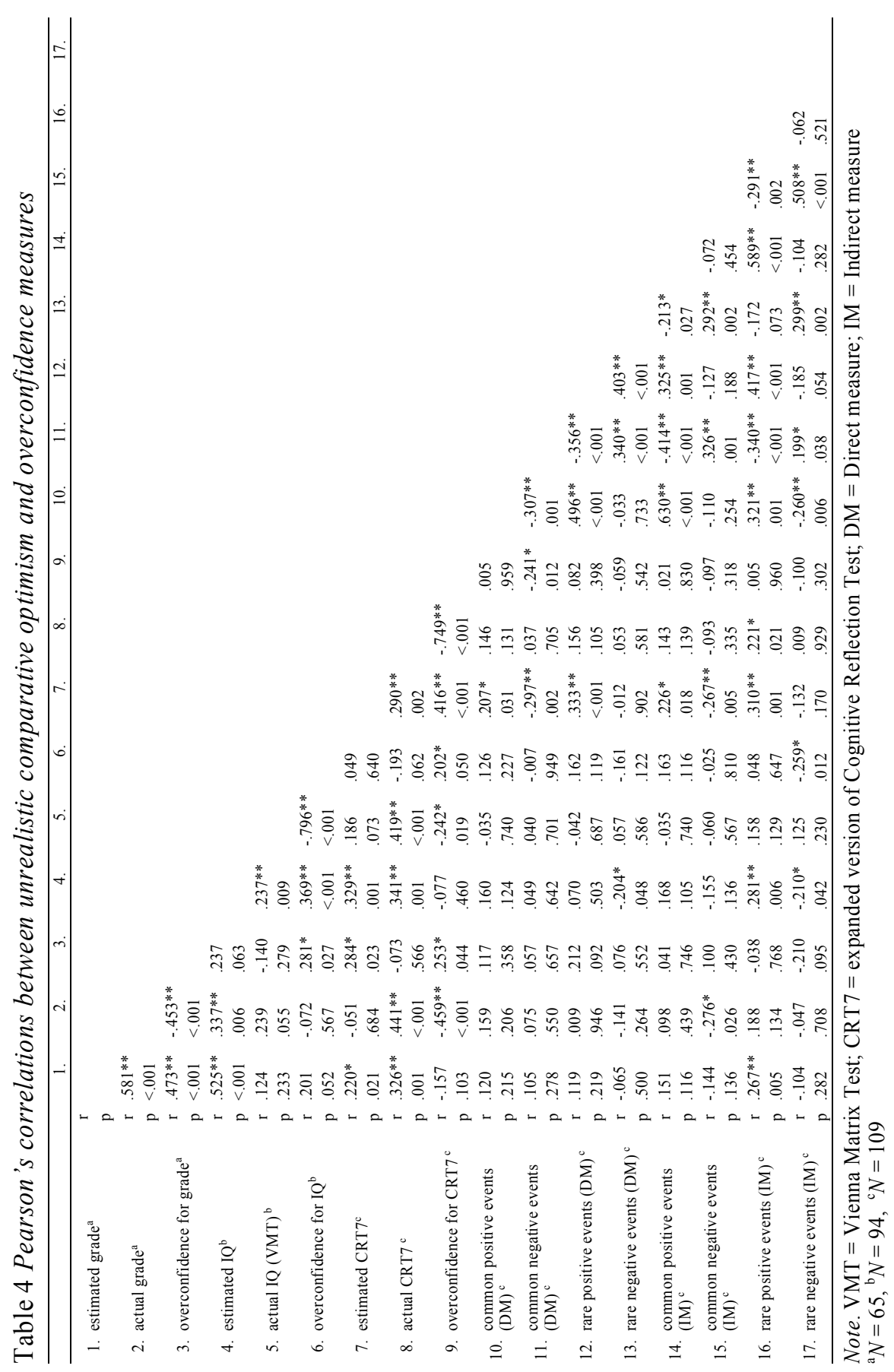


larger when the event was negative $(M($ common $)=-4.28, M($ rare $)=-4.50)$. When I compared direct and indirect estimates of likelihoods, I found 5 significant shifts in the opposite direction: owning a house ( $Z=$ $-4.790, p<.001)$, catching a flu in the next 4 years $(Z=-5.640, p<.001)$, being stressed before an exam $(Z=-5.044, p<.001)$, owning one's own island $(Z=-8.241, p<.001)$, and living past 100 years $(Z=-7.556, p<.001)$. Participants were significantly more optimistic regarding common positive events and also common negative events using the indirect measure. On the other hand, they displayed more optimism regarding rare negative events using the direct measure - they estimated much less likelihood of experiencing rare negative events when directly comparing their chances with the average student. The most pessimistic estimates were in estimating the likelihood of rare positive events using the direct measure - using the indirect measure, the pessimism almost disappeared and the participants judged their chances to be about the same as those of the average student.

\section{Discussion}

In this paper I tested three hypotheses: 1) whether participants will show optimism for common events, but sill show pessimism for rare events (regardless of their desirability), 2) whether there is a relationship between optimism and overconfidence and 3 ) whether indirect measure will yield more pessimism due to lower egocentrism.

The first hypothesis was not fully supported. The expected and observed results are shown in Table 5. The results of this study support neither the unrealistic hypothesis nor the egocentrism hypothesis fullyparticipants appeared to be somewhat pessimistic in estimating likelihoods of mainly positive events happening to them; they were quite optimistic in expecting to avoid negative events. Although I used the same tasks as Kruger and Burrus (2004) I did not

Table 5 Comparison of expected and observed results

\begin{tabular}{|c|c|c|c|c|}
\hline & $\begin{array}{l}\text { Expected } \\
\text { direction of } \\
\text { unrealistic } \\
\text { optimism } \\
\text { hypothesis }\end{array}$ & $\begin{array}{l}\text { Expected } \\
\text { direction of } \\
\text { statistical artefact } \\
\text { and egocentrism } \\
\text { hypotheses } \\
\text { (Kruger \& Burrus, } \\
\text { 2004) }\end{array}$ & $\begin{array}{l}\text { Observed } \\
\text { results by } \\
\text { direct measure }\end{array}$ & $\begin{array}{l}\text { Observed } \\
\text { results by } \\
\text { indirect } \\
\text { measure }\end{array}$ \\
\hline Common positive & + & + & - & + \\
\hline Common negative & - & + & - & - \\
\hline Rare positive & + & - & - & - \\
\hline Rare negative & - & - & - & - \\
\hline
\end{tabular}

Note. Positive/negative sign indicates whether the sum of likelihoods were greater $(+)$ or lower (-) for self than for the average student. Shaded areas represent optimistic expectation, blank areas represent pessimistic expectation (in comparison with average student). 
generally replicate their findings in my sample. In their study, participants showed optimism in all eight estimates of common positive events (they expected that they would be more likely than the average student in their class to experience them) and in all eight estimates of rare negative events (they expected that they would be less likely than the average student in their class to experience them). In general, they overestimated the probability of common events happening to them (regardless of their desirability) and underestimated the probability of rare events happening to them (again regardless of their desirability). In my sample there were only 3 out of 7 optimistic estimates of common positive events (and only two of them statistically significant), but participants still underestimated the likelihood of experiencing rare negative events and thus displayed the same optimism as Kruger and Burrus's sample. On the other hand, in my sample there were 5 out of 7 optimistic estimates of a common negative event. Thus, despite my predictions, based on Kruger and Burrus's study, my sample did not overestimate their likelihood of experiencing common negative results to the same extent.

However, closer scrutiny of the content of the questionnaire may explain some of these ambiguous results. Students in this sample were optimistic that they would have their own car, live past 70, and travel across Europe - events that are quite likely for the majority of future adults. Travelling across Europe meant probably something different for the participants in Kruger and Burrus's study, but it was regarded as a common positive event. Even though travelling abroad is very affordable nowadays, especially within Europe, it would be probably more suitable to use item "travel to the U.S." (or other con- tinent) for European samples. They were pessimistic in their lookouts for owning their own house, getting a prestigious award for their work, graduating with honors and having a starting salary of more than $€ 800$. These events are probably not so common for adults in Slovakia in the future compared with adults in the United States. Students at the Pedagogical Faculty know that if they are going to work as teachers, their salary will be low ${ }^{4}$ and there will be a small chance of them purchasing a house. Similarly, studying at the Pedagogical faculty is not considered prestigious so their degree results (and perhaps their expectations) are far lower compared with some other faculties.

On the other hand, current sample was also more optimistic in estimating common negative events - they were pessimistic only about catching the flu and being stressed out before an exam. They did not expect to get speeding tickets, to gain weight, to have a broken heart, to be involved in a car accident, or to undergo a painful procedure at the dentist. Some of these results can be explained by gender for example, women are usually safer drivers, so it is less likely that they will get a speeding ticket or be involved in a car crash. Also, students do not drive cars in Slovakia

\footnotetext{
${ }^{4}$ Salaries of teachers are calculated according to the tables provided by the Ministry of Education. Salary class is assigned according to the education degree and years of practice. Starting salary for a teacher with M.A. degree in education in his/her area of expertise with 0 years of practice belongs usually to the Salary Class 9. Starting salary in this category is $€ 613.50$ gross (Stupnica platových taríf pedagogických zamestnancov a odborných zamestnancov a zvýšenie platových tarif $v$ závislosti od dížky započitanej praxe účinné od 1. januára 2016, 2015), which is about $€ 550$ net.
} 
as much as they do in the U.S., so they consider car-related events as less likely for them when compared with American samples. Optimistic outlooks about one's appearance and not having one's heart broken are interesting in their own right and would need more examination, but they suggest that gender probably affects optimism bias. For example, White, Cunningham, and Titchener (2011) found that gender and experience both have several main effects in skill-related types of optimism among young novice drivers. Men displayed more optimism bias regarding their overall skills and hazard perception than women. Also Lin and Raghubir (2005) found that men are generally more unrealistically optimistic than women, which could partly explain the lower optimism of the participants.

The second hypothesis focused on the relationship between optimism and overconfidence. The results seem to support claims made by various researchers (Radcliffe \& Klein, 2002; Shepperd et al., 2013) that greater care should be taken in distinguishing between unrealistic comparative and unrealistic absolute optimism. For example, Radcliffe and Klein (2002) found that people high in comparative optimism possessed an adaptive risk and belief profile and knew more about risk factors for heart attacks, whereas people high in unrealistic absolute optimism exhibited the opposite pattern, showing some defensiveness about learning about the risk factors associated with heart attacks, although they were more at risk. Radcliffe and Klein (2002) used an objective measure to estimate the individual risks of a heart attack, but this is not always possible. Therefore, the next step should be to look for ways of distinguishing between adaptive and maladaptive forms of optimism (or to identify when optimism starts to become maladaptive and a defensive strategy).

The participants were overconfident in estimating their own performance in cognitive abilities tasks - the more overconfident they were, the worse their performance was. These findings are, in fact, consistent with the Thurstonian theory (Moore \& Healy, 2008), which holds that individuals with the best performances on any given task are most likely to underestimate their actual performances and also with Kruger-Dunning hypothesis (Kruger \& Dunning, 1999) that those most incompetent/unskilled lack the meta-cognitive ability to reflect upon their deficiencies. On the other hand, like other researchers (Armor \& Taylor, 2002; Taylor et al., 1992) I found moderate correlation between anticipated IQ and actual IQ score, and between anticipated performance in the CRT and actual performance. It means that people can evaluate their relative cognitive abilities, although they tend to highly overestimate the actual score. Intelligence was not much easier to estimate than the CRT the observed pattern of correlations was very similar for both intelligence and CRT. Both tests elicited high discrepancy between anticipated and actual performance, but despite this optimism, those who anticipated higher scores tended to score better than those expecting lower scores (though still much less than they anticipated). Overconfidence seems to be related more to the lack of insight or metacognitive skills, as it is consistently found that even people with high cognitive abilities often fail such relatively simple tasks as CRT, which require that a recognition of the first answer may be a wrong one (Čavojová \& Hanák, 2014; Frederick, 2005; Stanovich, 2011, 2012). 
Also the comparison of the three groups of participants in terms of their realistic/optimistic/pessimistic estimation of intelligence suggests that there is only a small overlap between comparative optimism and overconfidence - those who were most overconfident about their intelligence were not generally most optimistic in their estimations of the likelihood of experiencing positive events or avoiding negative events. It seems that optimism is quite context-relevant and probably depends on our previous experiences with the task (Armor \& Taylor, 2002), the framing of the task (Eiser et al., 2001) and other factors. Eiser et al. (2001) also suggest that exhibiting optimism bias can be affected by self-presentational strategies specific to the domain of exam performance that suppresses expressed optimism, and both our cognitive abilities tasks could be viewed as a sort of exam. It is probable that participants realized that their anticipated performance would be compared with their actual performance, which may lead them to potentially falsify boastful claims, although they still overestimated their abilities (Hardman, 2009).

The lack of correlation between overconfidence in the CRT and overconfident estimates of intelligence or optimistic bias in estimating future events suggests that optimism is domain-specific and that even such similar domains as cognitive tasks do not yield similar levels of overconfidence.

Another possible reason for the lack of relationships found between unrealistic comparative optimism and overconfidence could be that the sample appeared to be more pessimistic than optimistic in estimating likelihoods of various events happening to them. Moreover, the results comparing direct and indirect method do not conform to the observation made by Rose et al. (2008) that in- direct method, due the suppression of egocentrism bias, yields more pessimistic estimates. Quite the contrary, participants in this study were not unrealistically pessimistic when negative events were frequent.

As is true of many studies, this one also has some limits that should be mentioned. First, the sample used has its specifics (e.g., gender composition, career expectations, academic performance, etc.), therefore, the results are not generalizable to other populations, due to possible gender and other differences. The study also suffers the same methodological difficulties regarding the comparative optimism on an absolute level, such as the inability to know with whom the participants choose to compare themselves, or the apparent optimism or overconfidence may be due to statistical artefact reasons and may in fact reflect rational updating of beliefs (Benoit \& Dubra, 2011; Harris \& Hahn, 2011). Another limit may lay in the materials used - it would be necessary first to test the actual desirability and perceived frequency of events used in the questionnaire, as not all the positive events may be equally desirable for all participants (Carver et al., 2010).

\section{Conclusion}

To summarize the results: The participants in this study were generally pessimistic in their future prospects regarding the positive events happening to them, but on the other hand, they were generally optimistic that negative events are less likely to happen to them and this tendency was more pronounced for rare than for common events. The most probable explanation for this curious finding is that the study was done on a sample of pedagogy students, who may be less optimistic about their future prospects 
regarding high salary, recognition for work, owning a house, etc.. The problem of underpayment of teachers leading most able students to choose other than pedagogical professions (and leaving pedagogical faculties with students who are often below-average in their study results) is so notorious in Slovakia that it resulted in a strike of teachers earlier this year ${ }^{5}$. The results may also reflect some cultural differences in optimism/ pessimism, as the countries with more individualistic cultures tend to be more optimistic than countries with more collective cultures (Fischer \& Chalmers, 2008; Chang, 1996), and Slovakia lies somewhere in between (Bašnáková et al., 2016).

Also, there is only a small overlap between having overconfident estimates of one's own performance and optimistic future expectations. The lack of stronger correlation suggests that researchers should be more careful in distinguishing various manifestation of what is called optimistic bias or betterthan-average effect. Even in their influential paper, Taylor and Brown (1994) examine three manifestations of so called positive illusion: illusion of control, enhanced self-perceptions, and unrealistic optimism, which represent three distinct concepts, making it more difficult to test their claims (Asendorpf \& Ostendorf, 1998). Having more favorable picture of oneself and one's abilities and having unrealistic expectations from the future may not be so closely related after all. Moreover, as many critics of the concept of unrealistic optimism (Djulbegovic et al., 2011; Harris \& Hahn, 2011) and ungrounded overconfidence (Benoit \& Dubra, 2011; Olsson,

\footnotetext{
${ }^{5}$ The strike itself had only marginal effect on this study, if any, as the data were collected in 2014 .
}

2014) have noted, sometimes the researchers are too quick to ascribe "bias" to the participants. Since it is possible to estimate unrealistic absolute optimism only once we know the exact probabilities and risk factors for determining whether we are overly optimistic about our prospects, and we are far from perfectly knowledgeable beings (Gigerenzer, 2008; Gilovich, Griffin, \& Kahneman, 2002), it is probably rather unwise of researchers to treat all optimism found in the laboratory as unrealistic.

Received February 17, 2016

\section{References}

Armor, D. A., \& Taylor, S. E. (2002). When predictions fail: The dilemma of unrealistic optimism. In D. Gilovich, T. Griffin, \& D. Kahneman (Eds.), Heuristics and biases: The psychology of intuitive judgment (pp. 335-347). Cambridge, MA: Cambridge University Press.

Asendorpf, J. B., \& Ostendorf, F. (1998). Is selfenhancement healthy? Conceptual, psychometric, and empirical analysis. Journal of Personality and Social Psychology, 74(4), 955-966. doi:10.1037/0022-3514.74.4.955

Bašnáková, J., Brezina, I., \& Masaryk, R. (2016). Dimensions of culture: The case of Slovakia as an outlier in Hofstede's research. Československá Psychologie, 60(1), 13-25.

Benoit, J., \& Dubra, J. (2011). Apparent overconfidence. Econometrica, 79(5), 1591-1625. doi: 10.3982/ECTA8583

Carver, C. S., Scheier, M. F., \& Segerstrom, S. C. (2010). Optimism. Clinical Psychology Review, 30(7), 879-889. doi:10.1016/j.cpr.2010.01. 006

Colvin, C. R., \& Block, J. (1994). Do positive illusions foster mental health? An examination of the Taylor and Brown formulation. Psychological Bulletin, 116(1), 3-20. doi:10.1037/00332909.116.1.3

Colvin, C. R., Funder, D. C., \& Block, J. (1995). Overly positive self-evaluations and personality - negative implications for mental-health. Journal of Personality and Social Psychology, 68(6), 1152-1162. doi:10.1037//0022-3514.68.6.1152 
Correia, V. (n.d.). Sour illusions: What is adaptive about misbelief? Retrieved from https:// www.academia.edu/579448/Sour illusions What_is_adaptive_about_ili usional beliefs? auto $=$ download

Čavojová, V., \& Hanák, R. (2014). Assessing fluid rationality and its relation to cognitive styles. In T. Ahram, W. Karwowski, \& T. Marek (Eds.), Proceedings of the 5th International Conference on Applied Human Factors and Ergonomics AHFE 2014. Krakow.

Djulbegovic, B., Kumar, A., Magazin, A., Schroen, A. T., Soares, H., Hozo, I., ... \& Schell, M. J. (2011). Optimism bias leads to inconclusive results - an empirical study. Journal of Clinical Epidemiology, 64(6), 583-593. doi:10.1016/ j.jclinepi.2010.09.007

Eiser, J. R., Pahl, S., \& Prins, Y. R. A. (2001). Optimism, pessimism, and the direction of selfother comparisons. Journal of Experimental Social Psychology, 37(1), 77-84. doi:10.1006/ jesp. 2000.1438

Fischer, R., \& Chalmers, A. (2008). Is optimism universal? A meta-analytical investigation of optimism levels across 22 nations. Personality and Individual Differences, 45(5), 378-382. doi: $10.1016 /$ j.paid.2008.05.008

Frederick, S. (2005). Cognitive reflection and decision making. Journal of Economic Perspectives, 19(4), 25-42. doi:10.1257/ 089533005775196732

Gigerenzer, G. (2008). Rationality for mortals: How people cope with uncertainity? New York: Oxford University Press.

Gilovich, T., Griffin, D., \& Kahneman, D. (Eds.) (2002). Heuristics and biases: The psychology of intuitive judgment. New York: Cambridge University Press.

Hardman, D. (2009). Judgment and decision making. Psychological perspectives. London: BPS Blackwell.

Harris, A. J. L., \& Hahn, U. (2011). Unrealistic optimism about future life events: A cautionary note. Psychological Review, 118(1), 135-154. doi: $10.1037 / \mathrm{a} 0020997$

Heine, S. J. (1993). Cultural variation in unrealistic optimism, (August) M.A. Thesis, University of British Columbia.

Heine, S. J., \& Lehman, D. R. (1995). Cultural variation in unrealistic optimism: Does the West feel more invulnerable than the East? Journal of Personality and Social Psychology, 68(4), 595-607.
Henrich, J., Heine, S. J., \& Norenzayan, A. (2010). The weirdest people in the world? The Behavioral and Brain Sciences, 33, 61-83; discussion 83-135. doi:10.1017/S0140525X0999152X

Chambers, J. R., \& Windschitl, P. D. (2004). Biases in social comparative judgments: The role of nonmotivated factors in above-average and comparative-optimism effects. Psychological Bulletin, 130(5), 813-838. doi:10.1037/0033-2909. 130.5 .813

Chang, E. C. (1996). Evidence for the cultural specificity of pessimism in Asians vs. Caucasians: A test of a general negativity hypothesis. Personality and Individual Differences, 21(5), 819-822. doi:10.1016/0191-8869(96)00110-9

Klose, J., Černochová, D., \& Král, P. (2002). Videňský maticový test. Praha: Testcentrum.

Kolman, L., Noorderhaven, N. G., Hofstede, G., \& Dienes, E. (2003). Cross-cultural differences in Central Europe. Journal of Managerial Psychology, 18(1), 86-88. doi:10.1108/ 02683940310459600

Kruger, J., \& Burrus, J. (2004). Egocentrism and focalism in unrealistic optimism (and pessimism). Journal of Experimental Social Psychology, 40(3), 332-340. doi:10.1016/j.jesp.2003.06. 002

Kruger, J., \& Dunning, D. (1999). Unskilled and unaware of it: How difficulties in recognizing one's own incompetence lead to inflated self-assessments. Journal of Personality and Social Psychology, 77(6), 1121-1134. doi:10.1037/00223514.77.6.1121

Larrick, R. P., Burson, K. A., \& Soll, J. B. (2007). Social comparison and confidence: When thinking you're better than average predicts overconfidence (and when it does not). Organizational Behavior and Human Decision Processes, 102(1), 76-94. doi:10.1016/j.obhdp.2006.10. 002

Lench, H. C., \& Ditto, P. H. (2008). Automatic optimism: Biased use of base rate information for positive and negative events. Journal of Experimental Social Psychology, 44(3), 631-639. doi: 10.1016/j.jesp.2007.02.011

Lin, Y.-C., \& Raghubir, P. (2005). Gender differences in unrealistic optimism about marriage and divorce: Are men more optimistic and women more realistic? Personality and Social Psychology Bulletin, 31, 198-207. doi:10.1177/ 0146167204271325

Menon, G., Kyung, E. J., \& Agrawal, N. (2009). Biases in social comparisons: Optimism or pessi- 
mism? Organizational Behavior and Human Decision Processes, 108(1), 39-52. doi:10.1016/ j.obhdp.2008.05.001

Moore, D. a, \& Healy, P. J. (2008). The trouble with overconfidence. Psychological Review, 115 , 502-517. doi:10.1037/0033-295X.115.2.502

Norem, J. K., \& Chang, E. C. (2002). The positive psychology of negative thinking. Journal of Clinical Psychology. doi:10.1002/jclp.10094

Olsson, H. (2014). Measuring overconfidence: Methodological problems and statistical artifacts. Journal of Business Research, 67(8), 1766-1770. doi:10.1016/j.jbusres.2014.03.002

Radcliffe, N. M., \& Klein, W. M. P. (2002). Dispositional, unrealistic, and comparative optimism: Differential relations with the knowledge and processing of risk information and beliefs about personal risk. Personality and Social Psychology Bulletin, 28, 836-846. doi:10.1177 0146167202289012

Rose, J. P., Endo, Y., Windschitl, P. D., \& Suls, J. (2008). Cultural differences in unrealistic optimism and pessimism: The role of egocentrism and direct versus indirect comparison measures. Personality \& Social Psychology Bulletin, 34(9), 1236-1248. doi:10.1177/0146167208319764

Sharot, T. (2011). The optimism bias. Current Biology: $C B, 21(23)$, R941-945. doi:10.1016/ j.cub.2011.10.030

Shepperd, J. A., Carroll, P., Grace, J., \& Terry, M. (2002). Exploring the causes of comparative optimism. Psychologica Belgica, 42, 65-98 Retrieved from http://search.ebscohost.com/ login aspx ? direct $=$ true $\& d b=$ psyh $\& A N=2002$ 06881-007\&site=ehost-live \nshepperd@ufl. edu

Shepperd, J. A., Klein, W. M. P., Waters, E. A., \& Weinstein, N. D. (2013). Taking stock of unrealistic optimism. Perspectives on Psychological Science, 8(4), 395-411. doi:10.1177/ 1745691613485247

Scheier, M. F., \& Carver, C. S. (1985). Optimism, coping, and health: Assessment and implications of generalized outcome expectancies. Health Psychology: Official Journal of the Division of Health Psychology, American Psychological Association, 4(3), 219-247. Retrieved from http:// www.ncbi.nlm.nih.gov/pubmed/4029106

Stanovich, K. E. (2011). Rationality and the reflective mind. New York: Oxford University Press.
Stanovich, K. E. (2012). On the distinction between rationality and intelligence: Implications for understanding individual differences in reasoning. In K. J. Holyoak \& R. G. Morrison (Eds.), The Oxford Handbook of Thinking and Reasoning (pp. 343-365). Oxford: Oxford Handbooks Online. doi: 10.1093/oxfordhb/9780199734689. 013.0022

Stupnica platových taríf pedagogických zamestnancov a odborných zamestnancov a zvýšenie platových taríf v závislosti od dížky započítanej praxe účinné od 1. januára 2016 (2015). Ministry of Education, Science, Research, and Sport of the Slovak Republic. Retrieved from https://www. minedu.sk/data/att/9351.pdf

Sweldens, S., Puntoni, S., Paolacci, G., \& Vissers, M. (2014). The bias in the bias: Comparative optimism as a function of event social undesirability. Organizational Behavior and Human Decision Processes, 124(2), 229-244. doi:10. 1016/j.obhdp.2014.03.007

Taylor, S. E., \& Brown, J. D. (1988). Illusionand well-being: A social psychological perspective on mental health. Psychological Bulletin, 103(2), 193-210. doi:10.1037/0033-2909.103.2.193

Taylor, S. E., \& Brown, J. D. (1994). Positive illusions and well-being revisited: Separating fact from fiction. Psychological Bulletin, 116(1), 2127; discussion 28. doi:10.1037/0033-2909.116. 1.28

Taylor, S. E., Kemeny, M. E., Aspinwall, L. G., Schneider, S. C., Rodriguez, R., \& Hubert, M. (1992). Optimism, coping, psychological distress, and high-risk sexual behavior among men at risk for AIDS. Journal of Personality and Social Psychology, 63, 460-473.

Toplak, M. E., West, R. F., \& Stanovich, K. E. (2014). Assessing miserly information processing: An expansion of the Cognitive Reflection Test. Thinking \& Reasoning, 20(2), 147-168. doi:10.1080/13546783.2013.844729

Weinstein, N. D. (1980). Unrealistic optimism about future life events. Journal of Personality and Social Psychology, 39(5), 806-820. doi:10.1037/ 0022-3514.39.5.806

White, M. J., Cunningham, L. C., \& Titchener, K. (2011). Young drivers' optimism bias for accident risk and driving skill: Accountability and insight experience manipulations. Accident; Analysis and Prevention, 43(4), 1309-1315. doi: $10.1016 /$ j.aap.2011.01.013 